\title{
TRATAMENTO DA DOENÇA DE ALZHEIMER
}

\section{AVALIAÇÃO CRÍTICA SOBRE O USO DE ANTICOLINESTERÁSICOS}

\author{
OSVALDO P. ALMEIDA*
}

\begin{abstract}
RESUMO - A demência do tipo Alzheimer (DA) é uma doença degenerativa que há até pouco tempo não tinha nenhum tratamento eficaz. Pesquisas realizadas a partir da década de 70 revelaram que os sistemas cerebrais que utilizam acetilcolina encontram-se gravemente comprometidos nesses pacientes, o que serviu de base para a introdução da terapêutica de base colinérgica da doença. Os inibidores da acetilcolinesterase foram os primeiros medicamentos que demonstraram ser úteis para o tratamento da DA em ensaios clínicos controlados contra placebo. Este artigo revê os principais estudos desenhados para investigar a eficácia e efeitos adversos da tacrina, donepezil, rivastigmina, e metrifonato em pacientes com DA. Discutem-se, ainda, os fatores que podem interferir na resposta do paciente ao tratamento com anticolinesterásicos, bem como as limitações inerentes a esse tipo de abordagem terapêutica.
\end{abstract}

PALAVRAS-CHAVE: Alzheimer, tratamento, eficácia, efeitos adversos, efeitos colaterais, anticolinesterásico, acetilcolinesterase, acetilcolina, tacrina, donepezil, rivastigmina, metrifonato.

\section{Update on anticholinesterase treatment of Alzheimer's disease}

ABSTRACT - Alzheimer's disease (AD) is a neurogenerative disorder that had no effective treatment until a few years ago. Many studies published during the past 20 years showed that the disease is associated with the disruption of brain systems that use acetilcholine. These findings shaped the development of the cholinergic treatment strategies for $\mathrm{AD}$. The cholinesterase inhibitors were the first class of drugs to show positive results in doubleblind placebo controlled studies for the treatment of AD. This paper reviews efficacy and safety studies of tacrine, donepezil, rivastigmine, and metrifonate in patients with AD. Factors that may interfere with patients' response to treatment are also discussed, as well as the limitations associated with the use of this class of drugs.

KEY WORDS: Alzheimer, treatment, efficacy, adverse effects, side effects, anticholinesterase inhibitors, acetilcholine, tacrine, donepezil, rivastigmine, metrifonate.

A doença de Alzheimer (DA) tem sido associada a uma série de alterações genéticas, neuropatológicas, e neurofisiológicas ${ }^{1}$. As anormalidades nos sistemas cerebrais que utilizam acetilcolina são consideradas características da doença e incluem: (1) redução desproporcionalmente grande na quantidade de acetilcolina ${ }^{2,3}$; (2) redução de colina-acetiltransferase ${ }^{2,3}$; (3) degeneração do núcleo basal de Meynert ${ }^{4,5}$; (4) alteração no número e sensibilidade de receptores nicotínicos e muscarínicos cerebrais $^{6,7}$ e; (5) sensibilidade aumentada aos efeitos de drogas anticolinérgicas como a escopolamina ${ }^{6}$. Além disso, ocorre comprometimento na expressão do RNA mensageiro de receptores nicotínicos de neurônios contendo a forma hiperfosforilada da 'tau', sugerindo que as disfunções no sistema de transporte intracelular típicas da DA interferem na formação de receptores colinérgicos ${ }^{7}$.

*Unidade de Idosos do Departamento de Saúde Mental da Santa Casa de São Paulo. O autor é parcialmente financiado pelo CNPq (Pesquisador nível IIa). Aceite: 25-maio-1998.

Dr. Osvaldo P. Almeida - Department of Psychiatry and Behavioural Science - Queen Elizabeth II Medical Centre - Nedlands-Perth, WA 6009 - Australia. FAX: 618 93463828. E-mail:osvalm@cyllene.uwa.edu.au 
Várias abordagens terapêuticas foram desenvolvidas ao longo dos últimos 20 anos com o objetivo de otimizar o funcionamento do sistema colinérgico dos portadores de DA. A estratégia que se mostrou mais eficaz até o momento foi a da inibição da acetilcolinesterase, enzima responsável pela degradação da acetilcolina. Este artigo apresenta breve revisão sobre os principais estudos que investigaram a eficácia e segurança dos anticolinesterásicos no tratamento de pacientes com DA. A discussão será restrita apenas àquelas drogas que já se encontram disponíveis no mercado para tratamento da DA ou que estejam em fase final de aprovação para comercialização.

\section{Tacrina}

O potencial da tacrina para tratamento da DA foi inicialmente descrito por Summers e col. em $1986^{8}$. Após esse primeiro relato vários estudos controlados foram realizados com a finalidade de verificar a real eficácia e segurança da tacrina no tratamento de pacientes com DA. Em 1994 Knapp e col. ${ }^{9}$ publicaram o ensaio clínico mais completo até o momento investigando a eficácia da tacrina. Um total de 653 pacientes foram divididos em 4 grupos: (1) placebo, (2) tacrina até $80 \mathrm{mg} / \mathrm{dia}$, (3) tacrina até $120 \mathrm{mg} / \mathrm{dia}$ e, (4) tacrina até $160 \mathrm{mg} / \mathrm{dia}$. A idade média dos pacientes era de 72,8 anos e o escore médio no Mini-Exame do Estado Mental (MMSE) era 18,7. A Fig 1 resume os resultados da evolução dos escores no MMSE e 'Alzheimer's Disease Assessment Scale - cognitive section' (ADAS-cog) ao longo das 30 semanas de tratamento. Os pacientes recebendo tacrina na dose de $160 \mathrm{mg} / \mathrm{dia}$ apresentaram uma melhora cognitiva discreta porém significativa em relação ao grupo placebo, conforme demonstrado pela evolução dos escores no ADAS-cog e MMSE. Além disso, a melhoria do desempenho cognitivo estava associada a doses mais elevadas da medicação (resposta/dose para ADAS-cog, $\mathrm{p}=0,004$; MMSE, $\mathrm{p}=0,008$ ).

Um dado que chama atenção nesse estudo é o alto índice de abandono do tratamento: apenas 40,3\% dos pacientes completaram as 30 semanas de acompanhamento. O aumento da dose estava associado a número crescente de abandonos, de forma que só $26,9 \%$ dos 238 pacientes alocados para receberem $160 \mathrm{mg} / \mathrm{dia}$ de tacrina chegaram ao final do ensaio clínico. Em outras palavras, a dose que se mostrou mais eficaz para o tratamento da DA só foi tolerada por aproximadamente $1 / 4$ dos pacientes.

Os efeitos colaterais mais freqüentemente observados com o uso de tacrina são náuseas/vômitos (28\%) e diarréia $(16 \%)^{10}$, embora a complicação mais temida seja a elevação das transaminases hepáticas (hepatite medicamentosa). Watkins e col. ${ }^{11}$ monitoraram as transaminases hepáticas de 628 pacientes recebendo tacrina, dos quais apenas 84 completaram o seguimento de 30 semanas. A Fig 2 mostra a porcentagem de indivíduos com elevação 3 ou mais vezes maior do que o limite superior considerado normal da transaminase pirúvica (TGP). O período entre 4 e 12 semanas é aquele de maior risco para o desenvolvimento de hepatite (Fig 2). De fato, mais de $90 \%$ dos indivíduos que apresentam elevação das transaminases o fazem dentro das primeiras 12 semanas de tratamento ${ }^{10}$. Essa elevação é na maior parte das vezes transitória, com os níveis séricos retornando ao normal ao longo das semanas subsequentes com o decréscimo da dose $(40 \mathrm{mg} /$ dia de redução até que haja queda nos níveis de TGP) ${ }^{11}$.

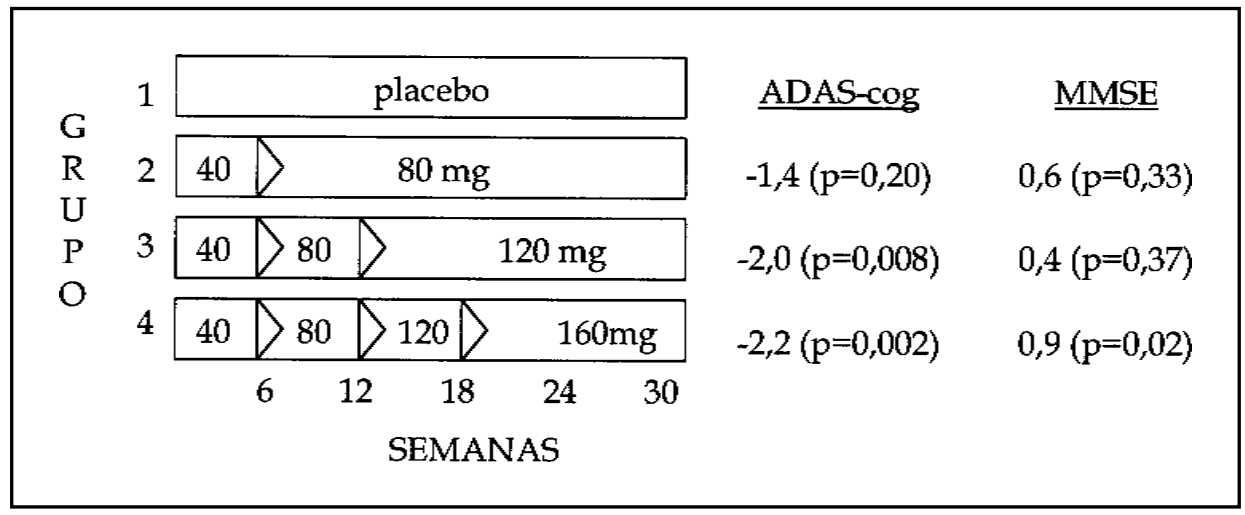

Fig 1. Desenho de estudo para administração de tacrina ao longo de 30 semanas. As duas colunas à direita mostram a variação média nos escores do ADAS-cog e do MMSE em relação ao grupo placebo. Os valores de $p$ referem-se à análise de intenção para tratar (Adaptado a partir de Knapp et al., 1994). 


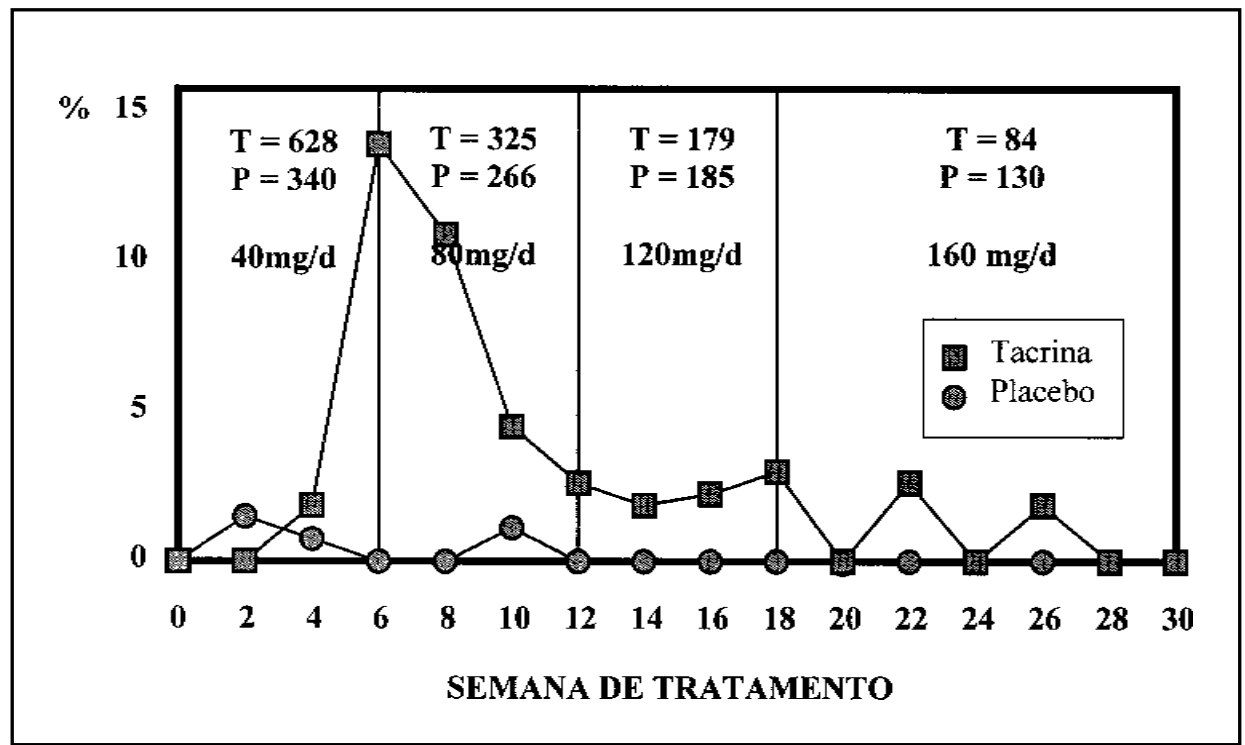

Fig 2. Probabilidade condicional de desenvolver elevação de 3 ou mais vezes no limite superior da TGP. A figura representa pacientes recebendo terapia contínua ao longo dos respectivos períodos. ' $T$ ' representa o número de pacientes recebendo tacrina e 'P' placebo (Adaptado a partir de Watkins et al., 1994) ${ }^{11}$.

Knopman e col. ${ }^{12}$ investigaram o impacto da utilização de tacrina sobre a mortalidade e necessidade de institucionalização durante seguimento clínico de 2 anos. Os pacientes acompanhados neste estudo eram os mesmos que haviam sido avaliados por Knapp e col. ${ }^{9}$. Indivíduos recebendo tacrina em doses maiores que 80 $\mathrm{mg}$ /dia apresentavam chance 2,7 vezes menor de serem institucionalizados que aqueles tratados com dose inferior a $40 \mathrm{mg} / \mathrm{dia}$ ou placebo. Além disso, observou-se uma tendência para menor mortalidade entre os pacientes tratados inicialmente com doses de tacrina entre 120 e $160 \mathrm{mg} / \mathrm{dia}$. O conjunto desses achados sugere que a utilização de doses adequadas de tacrina no tratamento de portadores de DA pode ter implicações sobre a evolução da doença a longo prazo.

Além disso, há evidências de que o uso de tacrina no tratamento de pacientes com DA pode reduzir os custos associados à doença. Wimo e col. ${ }^{13}$ construíram um modelo de sobrevivência para a população sueca de portadores de DA, levando em consideração o fato de que a institucionalização desses indivíduos pode ser influenciada pelo uso de tacrina ${ }^{12}$. Eles estimaram que pacientes com escores no MMSE entre 10 e 24 (52\% do total dos pacientes com DA) e tratados com $160 \mathrm{mg}$ diários de tacrina apresentariam uma melhora média de 2,6 pontos no MMSE. O impacto clínico dessa melhora representaria uma redução de 1,3\% nos custos associados ao acompanhamento de toda a população sueca com DA. Em outras palavras, o uso de tacrina parece ter efeitos benéficos, embora modestos, sobre os custos associados à DA.

\section{Donepezil}

O donepezil foi a segunda medicação aprovada nos Estados Unidos para o tratamento da DA. O estudo que serviu de base para a introdução da droga no mercado americano foi publicado por Rogers e col. em $1996^{14}$. O donepezil é um anticolinesterásico que apresenta meia-vida aproximada de 70 horas (em contraste com as 3,5 horas da tacrina ${ }^{10}$ ) e que se mostrou eficaz no tratamento da DA em doses de 5 e $10 \mathrm{mg}$, mas não para doses de 1 ou $3 \mathrm{mg}$ diários ${ }^{14}$.

Recentemente, Rogers e col. ${ }^{15}$ ampliaram os resultados iniciais sobre a eficácia e segurança do donepezil no tratamento de pacientes com DA. Os pacientes foram randomicamente distribuídos para receberem placebo $(\mathrm{n}=162), 5 \mathrm{mg}$ de donepezil $(\mathrm{n}=154)$, ou $10 \mathrm{mg}$ de donepezil $(\mathrm{n}=157)$ durante 24 semanas. A Fig 3 mostra a evolução nos escores do ADAS-cog e MMSE ao longo do tratamento. Pacientes recebendo a droga ativa apresentaram melhora significativa do desempenho cognitivo quando comparados ao grupo placebo, embora não se observasse diferença significativa entre aqueles que receberam 5 e $10 \mathrm{mg}$ de donepezil. Ao final de 24 


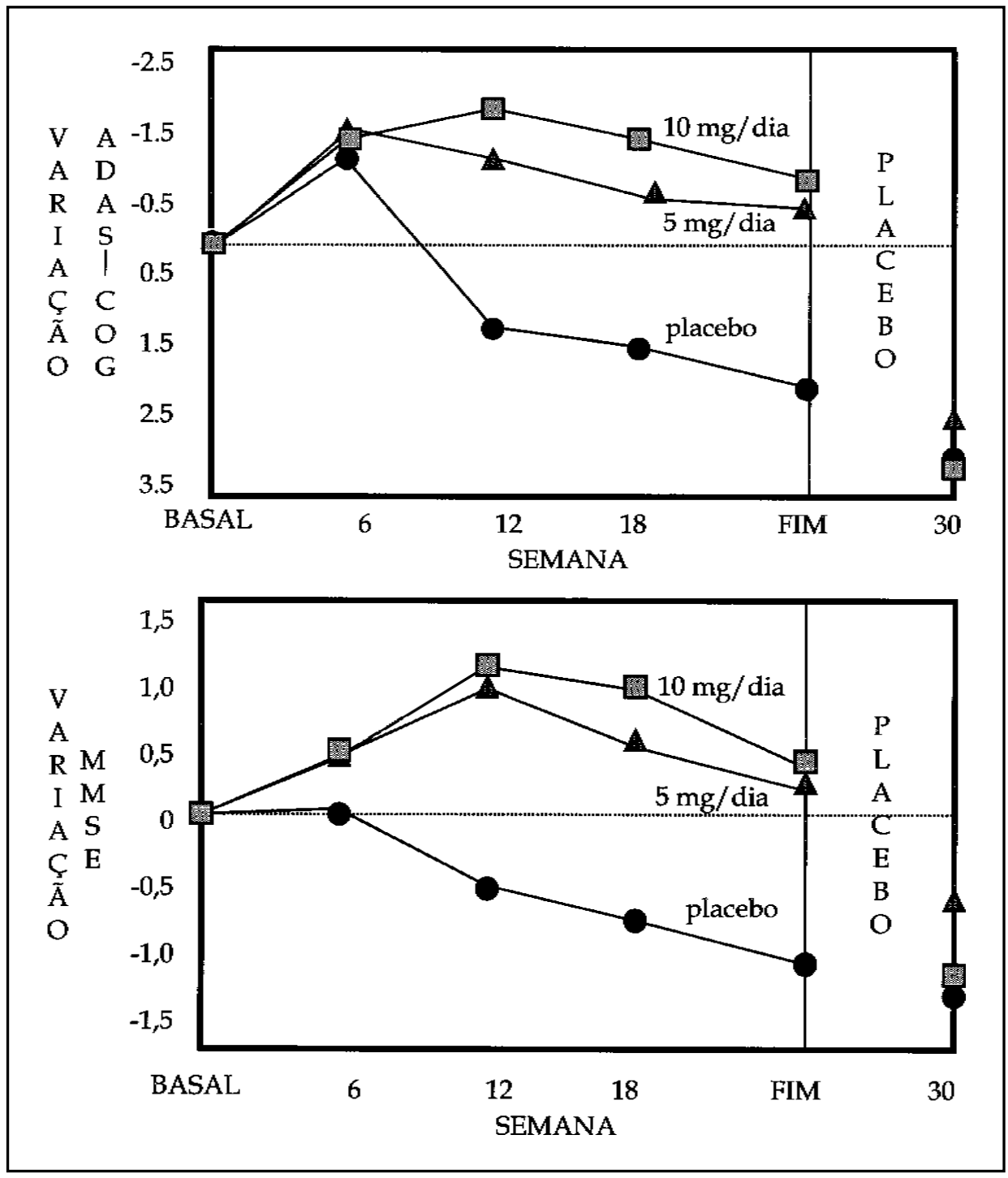

Fig 3. Variação nos escores do ADAS-cog e MMSE em pacientes com DA recebendo donepezil. Tratamento ativo com 5 ou $10 \mathrm{mg}$ de donepezil produziu melhora cognitiva em ambos os grupos quando comparados a pacientes recebendo placebo. Entre as semanas 24 (FIM) e 30 foi realizado 'washout' com placebo (Adaptado a partir de Rogers et al., 1998) $)^{15}$.

semanas de tratamento a medicação foi suspensa e uma nova avaliação realizada após 6 semanas. A vantagem observada entre os pacientes recebendo donepezil em relação aos controles utilizando placebo desapareceu na semana 30, sugerindo que a medicação não interfere com a evolução natural da doença.

Os efeitos adversos observados entre os pacientes desse ensaio clínico aparecem resumidos na Tabela 1. Indivíduos recebendo $10 \mathrm{mg} /$ dia de donepezil relataram significativamente mais sintomas como diarréia, náusea, vômito e cãibras. Esses sintomas são, em sua maioria, temporários e tendem a remitir com a evolução do tratamento $^{14}$. Além disso, o donepezil parece ser bem tolerado pela maioria dos pacientes e foi apenas ocasionalmente associado a descontinuação do tratamento: $85 \%$ e $68 \%$ respectivamente dos doentes que receberam 
Tabela 1. Porcentagem de efeitos adversos decorrentes do tratamento com placebo ou donepezil entre pacientes com doença de Alzheimer

\begin{tabular}{cccc}
\hline Efeitos adversos & $\begin{array}{c}\text { Placebo } \\
\mathrm{n}=162(\%)\end{array}$ & $\begin{array}{c}\text { Donepezil }(\%) 5 \mathrm{mg} / \mathrm{dia} \\
\mathrm{n}=154\end{array}$ & $\begin{array}{c}\text { Donepezil } 10 \mathrm{mg} / \mathrm{dia} \\
\mathrm{n}=157(\%)\end{array}$ \\
\hline Fadiga & 2 & 5 & $8^{*}$ \\
Diarréia & 7 & 9 & $17^{*}$ \\
Náusea & 4 & 4 & $17^{*}$ \\
Vômito & 2 & 3 & $10^{*}$ \\
Anorexia & 2 & 2 & 7 \\
Cãibras & 1 & 6 & $8^{*}$ \\
Tontura & 4 & 10 & 8 \\
Rinite & 2 & 1 & 6 \\
\hline
\end{tabular}

*Taxa significativamente maior ( $\mathrm{p}<0,05)$ que a observada no grupo placebo. (Adaptado a partir de Rogers et al., 1998) ${ }^{15}$.

5 e $10 \mathrm{mg}$ de donepezil completaram as 30 semanas de acompanhamento ( $80 \%$ no caso do grupo placebo) ${ }^{15}$. Assim sendo, o conjunto desses dados indica que a dose de $5 \mathrm{mg}$ diários de donepezil é a que produz o melhor balanço entre eficácia e tolerância.

Rogers e seus colaboradores ${ }^{14}$ estimaram, também, que a utilização de $10 \mathrm{mg}$ diários de donepezil retarda a deterioração cognitiva em aproximadamente 40 semanas. Ou seja, os escores de pacientes recebendo donepezil em um determinado momento são semelhantes aos escores de indivíduos recebendo placebo 40 semanas antes.

\section{Rivastigmina}

A rivastigmina (ENA-713) é um novo anticolinesterásico que vem sendo testado quanto à sua eficácia e tolerabilidade para o tratamento de pacientes com doença de Alzheimer. Esse medicamento já recebeu aprovação para comercialização no Brasil e Suíça, mas ainda aguarda o parecer do FDA para que o mesmo seja introduzido no mercado norte-americano. Estudos com animais de laboratório indicam que a rivastigmina inibe preferencialmente a acetilcolinesterase cerebral ${ }^{16}$. Além disso, a droga parece atuar de forma adequada no hipocampo e córtex cerebral, que são áreas significativamente comprometidas em pacientes com doença de Alzheimer ${ }^{16}$.

Os dados disponíveis até o momento quanto à eficácia clínica da rivastigmina ainda são preliminares. Anand e col. ${ }^{17}$ relataram os achados referentes a 516 pacientes tratados com o medicamento. Doses entre 6 e 12 mg diários produziram melhora clínica de acordo com o "Clinical Global Impression of Change" (CGIC) e em alguns subtestes cognitivos como a "Substituição de Dígitos por Símbolos"17. Os efeitos adversos mais comuns associados ao uso de rivastigmina foram cefaléia, náusea, tontura e diarréia ${ }^{17}$.

Infelizmente, a quantidade limitada de informações disponíveis até o momento sobre a rivastigmina impede que se tenha idéia clara quanto à sua real eficácia e segurança no tratamento de pacientes com DA.

\section{Metrifonato}

O metrifonato é um inibidor irreversível da acetilcolinesterase. A eficácia dessa droga no tratamento de pacientes com DA foi recentemente descrita em um estudo pequeno publicado por Becker e col. ${ }^{18}$. Eles avaliaram um total de 47 pacientes, dos quais 23 receberam a droga ativa e 24 placebo por 6 meses. A dose do medicamento foi individualizada de acordo com a massa corpórea dos pacientes: $2 \mathrm{mg} / \mathrm{Kg}$ de peso nos primeiros 5 dias, 0,95 $\mathrm{mg} / \mathrm{Kg}$ no dia 6 , e 2,9 mg/Kg semanais a partir do dia 7 . Esses níveis foram considerados adequados para induzir a inibição de 50-70\% da acetilcolinesterase das hemácias. Análise de covariância utilizando os escores iniciais como covariáveis revelou que os pacientes recebendo metrifonato apresentavam discreta melhora nos escores do ADAS-cog, enquanto aqueles tratados com placebo exibiam sinais de deterioração cognitiva ao longo do estudo $(\mathrm{p}<0,01)$. Eles estimaram, ainda, que a taxa anual de declínio no grupo utilizando a droga ativa era de 1,1 pontos, o que se comparava favoravelmente com os 6,9 pontos do grupo recebendo placebo. O mesmo tipo de resposta 


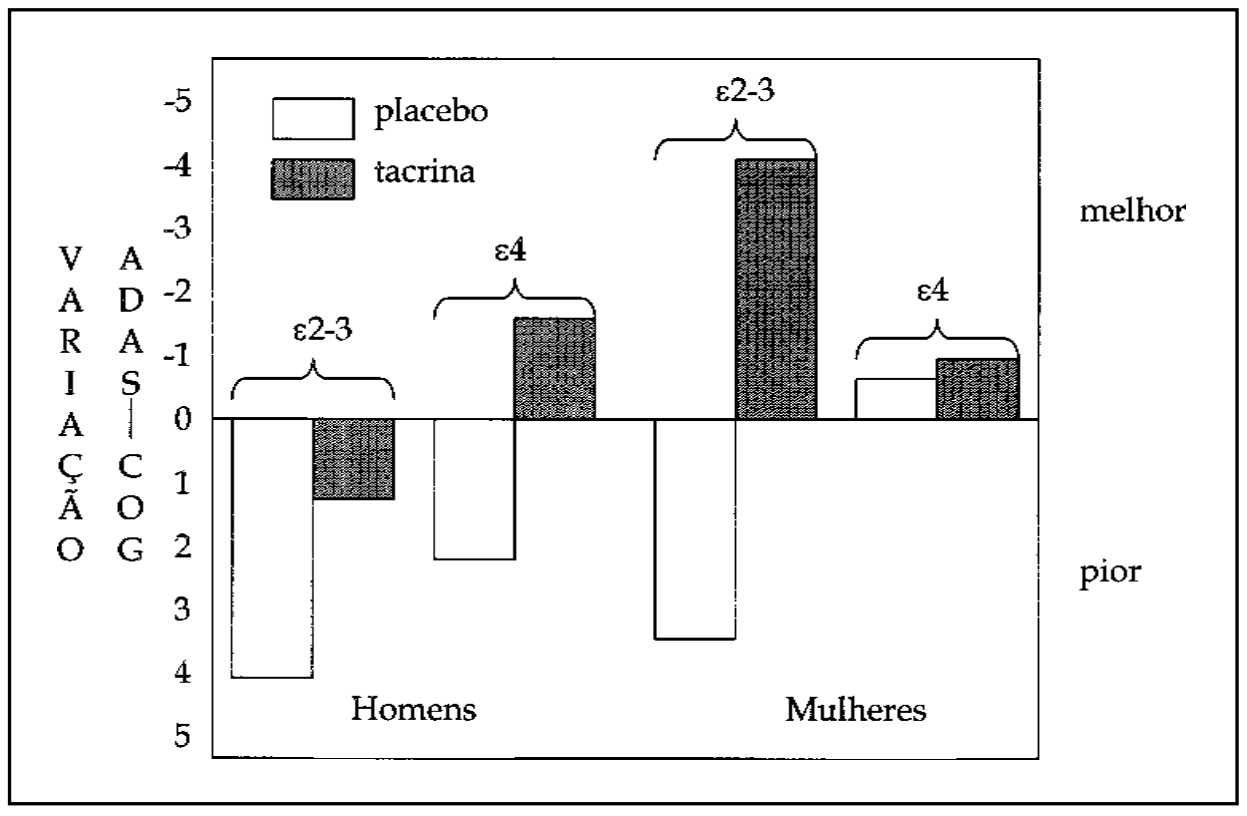

Fig 4. Variação média dos escores no ADAS-cog (escore após 30 semanas de tratamento-escore imediatamente antes do início do tratamento) de acordo com sexo e tipagem da apolipoproteína E. Os resultados incluem pacientes recebendo tacrina na dose de 80, 120, e $160 \mathrm{mg}$ diários (Adaptado a partir de Farlow et al., 1998) ${ }^{21}$.

foi observado quando se utilizou o MMSE como medida de eficácia, embora a diferença entre o grupo placebo e metrifonato não tenha atingido significância estatística nesse caso específico $(\mathrm{p}=0,09)$.

Quatorze eventos adversos foram observados nesse estudo em associação com o uso de metrifonato, embora estes não tenham sido considerados suficientemente graves para provocar mudanças no regime terapêutico. Becker e seus colaboradores ${ }^{18}$ tampouco observaram alterações significativas de sinais vitais, exames laboratoriais, ou eletrocardiograma entre os pacientes tratados com metrifonato. Entretanto, a apresentação dos resultados sobre efeitos adversos nesse estudo limitou-se a afirmações genéricas e compromete uma avaliação mais completa sobre os efeitos adversos provocados pela droga.

\section{FATORES QUE INFLUENCIAM A RESPOSTA AOS ANTICOLINESTERÁSICOS}

Uma avaliação geral dos estudos que utilizaram tacrina para o tratamento de pacientes com DA não encontrou nenhuma influência de sexo ou idade sobre a resposta terapêutica ${ }^{19}$. Pacientes com quadro clínico de gravidade moderada parecem ser os que mais se beneficiam com o uso da medicação, enquanto aqueles com afasias graves praticamente não respondem ${ }^{19}$.

\section{Apolipoproteína $E$}

Poirier e col..$^{20}$ desenharam um estudo para investigar o impacto da apolipoproteína E (ApoE) sobre a resposta terapêutica. Vinte e dois dos 40 pacientes avaliados eram portadores do alelo e4 da apolipoproteína E. Esses pacientes apresentavam redução na atividade da colina-acetiltransferase e pior resposta terapêutica à tacrina em ensaio clínico com duração de 30 semanas. De fato, mais de $80 \%$ dos pacientes que não possuíam o alelo e 4 apresentaram melhora significativa na escala ADAS, enquanto $60 \%$ dos portadores de e4 sofriam deterioração nesses mesmos escores.

Em contraste com os achados acima descritos, Odozze e col. ${ }^{21}$ observaram que $71 \%$ dos 67 pacientes e4+ tratados com tacrina por 6 meses (dose até $160 \mathrm{mg} /$ dia) apresentaram melhora das dificuldades cognitivas, enquanto apenas $34 \%$ dos 35 pacientes e4- foram considerados respondedores. 


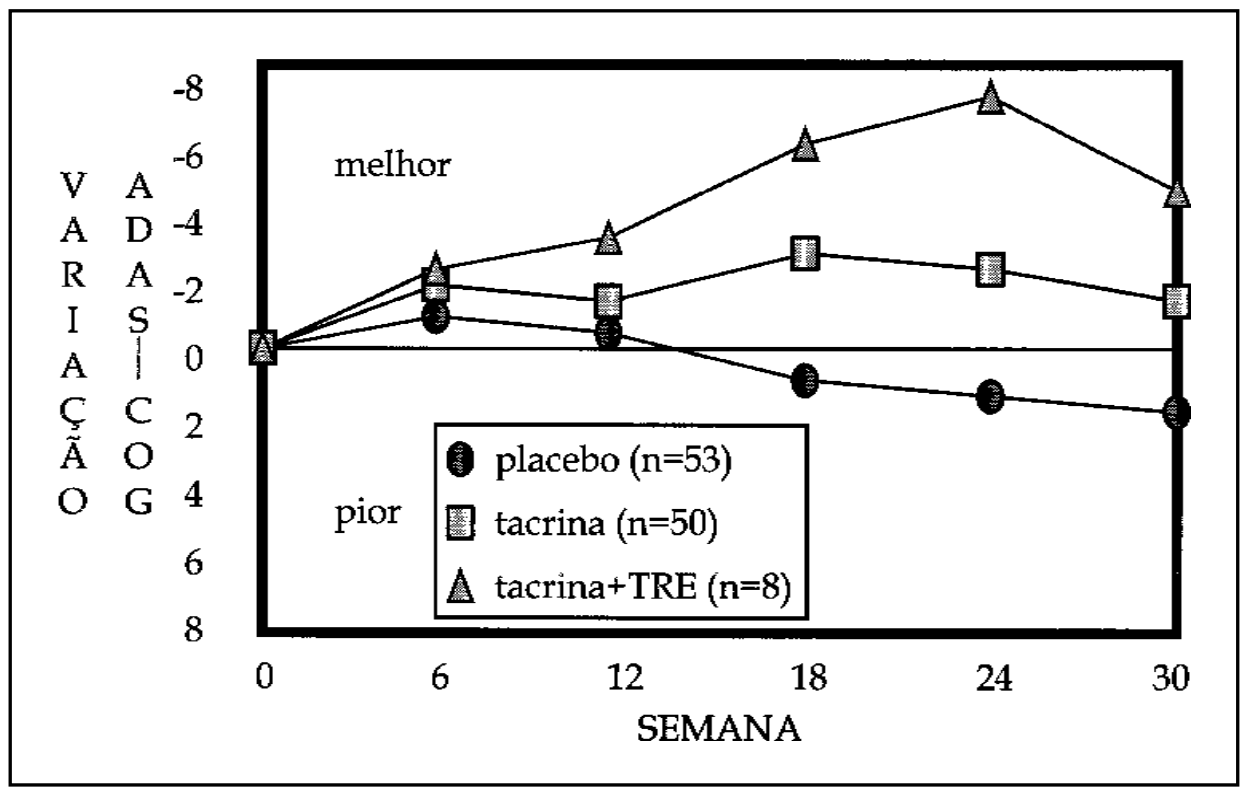

Figura 5. Variação nos escores da ADAS-cog entre pacientes do sexo feminino com doença de Alzheimer recebendo placebo, tacrina, ou tacrina + terapia de reposição estrogênica (TRE) (Adaptado a partir de Schneider et al., 1996$)^{27}$.

A aparente discrepância entre esses dois estudos pode ser parcialmente explicada pela interação entre tipagem genética da ApoE e sexo. Farlow e col. ${ }^{22}$ investigaram o impacto da ApoE e sexo sobre a resposta ao tratamento com tacrina em 528 pacientes com doença de Alzheimer ${ }^{22}$. Eles observaram que a genotipagem da apolipoproteína $\mathrm{E}$ era, na realidade, insuficiente para explicar a resposta ao tratamento. De fato, o sucesso do tratamento parece depender de uma interação complexa entre genotipagem da ApoE e sexo (Fig 4).

\section{Estrógeno}

Vários estudos publicados recentemente indicam que a terapia de reposição estrogênica está associada a redução no risco de desenvolvimento de $\mathrm{DA}^{23-26}$. Schneider e col. ${ }^{27}$ decidiram, então, investigar de forma retrospectiva o impacto da reposição estrogênica sobre a resposta terapêutica à tacrina em mulheres com DA. Das 111 pacientes disponíveis para análise 53 receberam placebo, 50 receberam tacrina, e 8 receberam tacrina + placebo por 30 semanas. A Fig 5 ilustra o desempenho dessas pacientes na ADAS-cog ao longo do tempo. Pacientes recebendo tacrina apresentaram melhor evolução do que as pacientes tratadas com placebo, sendo que as pacientes tratadas com tacrina+estrógeno apresentavam o melhor perfil de resposta terapêutica entre as respondedoras. É evidente que o número de pacientes recebendo estrógeno+tacrina nesse estudo era muito pequeno e limita a generalização desses achados.

\section{CONCLUSÃO E PERSPECTIVAS}

Há até poucos anos o diagnóstico de Doença de Alzheimer era acompanhado de intenso niilismo por parte dos médicos quanto às possibilidades de tratamento. A recente introdução de drogas anticolinesterásicas como a tacrina e donepezil deu ao médico a possibilidade de intervir farmacologicamente sobre os sintomas cognitivos característicos da doença, embora os benefícios associados ao uso desses medicamentos ainda seja muito limitado. E é fácil entender porque o atual 
tratamento da DA é considerado insatisfatório. A DA provoca degeneração de diversos sistemas neurais além dos que utilizam acetilcolina, o que sugere ser pouco provável que a reposição de um único neurotransmissor seja suficiente para provocar melhora sintomatológica significativa ${ }^{28}$. Além disso, praticamente $60-70 \%$ das células do núcleo basal de Meynert já degeneraram quando os sintomas clínicos da doença se manifestam ${ }^{4,28}$ — quão eficaz pode ser um tratamento que age sobre um substrato anatômico progressivamente mais escasso?

Tratamentos experimentais vêm sendo testados com o objetivo de desenvolver drogas capazes de interferir com o processo fisiopatológico básico da doença. Os candidatos naturais até o momento são os mecanismos envolvendo a fosforilação da $\operatorname{tau}^{29}$ e a deposição de $\beta$-amilóide ${ }^{30}$. O uso de anticolinesterásicos em pacientes com DA representa apenas o primeiro passo na busca de um tratamento capaz de modificar a evolução natural e, se possível, impedir a manifestação clínica da doença $\mathrm{a}^{31}$.

Nota - Dois novos estudos investigando a eficácia e tolerabilidade do metrifonato foram publicados desde a submissão desta revisão crítica. Cummings e col. ${ }^{32}$ estudaram um total de 480 pacientes com DA que foram alocados para receber placebo ou doses baixas, médias e elevadas de metrifonato (até $60 \mathrm{mg} / \mathrm{dia}$ ) por 30 semanas. Após 3 meses de tratamento, os pacientes recebendo metrifonato apresentavam vantagem de 2,94 pontos em relação àqueles tratados com placebo (intervalo de confiança de $95 \%=1,61$ a 4,27) na ADAS-cog. Indivíduos tratados com as doses mais elevadas de metrifonato foram os que apresentaram a melhor resposta terapêutica. A droga foi bem tolerada pelos pacientes ( $91 \%$ completaram as 30 semanas de tratamento), sendo os principais efeitos colaterais diarréia, dores abdominais e náuseas. O outro estudo, publicado por John Morris e seus colaboradores $^{33}$, revelou que além dos efeitos benéficos sobre a cognição o metrifonato também contribuiu para melhorar o comportamento dos pacientes, conforme avaliação com o "Neuropsychiatric Inventory - NPI" ( $p=0,016)$. Esses resultados indicam que o metrifonato tem um perfil de eficácia e tolerabilidade semelhante ao donepezil. Além disso, eles confirmam os resultados

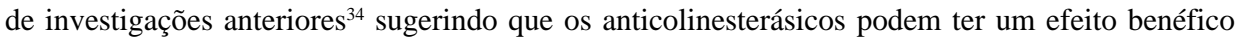
sobre o comportamento de pacientes com DA.

\section{REFERÊNCIAS}

1. Almeida OP. Biologia molecular da doença de Alzheimer: Uma luz no fim do túnel? Rev Assoc Méd Bras 1997;43:77-81.

2. Katzman R. Alzheimer's disease. N Engl J Med 1986;314:964-973.

3. Nordberg A, Carlson LA, Winblad B. Biological markers and the cholinergic hypothesis in Alzheimer's disease. Acta Neurol Scand 1992;85:54-58.

4. Whitehouse PJ, Price DL, Struble RG, Clarck AW, Coyle JT, DeLong MR. Alzheimer's disease and senile dementia: loss of neurons in the basal forebrain. Science 1982; 215:1237-1239.

5. Coyle JT, Price DL, DeLong MR. Alzheimer's disease: a disorder of cholinergic innervation. Science 1983;219:1184-1190.

6. Newhouse PA. Alzheimer's disease and the cholinergic system: an introduction to clinical pharmacological research. In Heston LL (ed). Progress in Alzheimer's disease and similar conditions. Washington DC: American Psychiatric Press, 1997:213-231.

7. Schröder H, Wevers A. Nicotinic aceylcoline receptors in Alzheimer's disease. Alzheimer's Dis Rev 1998;3:20-27.

8. Summers WK, Majovski LV, Marsh GM, Tachiki K, Kling A. Oral tetrahydroaminoacridine in long-term treatment of senile dementia Alzheimer type. N Engl J Med 1986;315:1241-1245.

9. Knapp MJ, Knopman DS, Solomon PR, Pendlebury WW, Davis CS, Gracon SI. A 30-Week randomized controlled trial of hight-dose tacrine in patients with Alzheimer's disease. J Am Med Assoc 1994;271:985-991.

10. Davis KL, Powchik P. Tacrine. Lancet 1995;345:625-630.

11. Watkins PB, Zimmerman HJ, Knapp MJ, Gracon SI, Lewis KW. Hepatotoxic effects of tacrine administration in patients with Alzheimer's disease. J Am Med Assoc 1994;271:992-998.

12. Knopman D, Schneider L, Davis K, et al. Long-term tacrine (Cognex) treatment: effects on nursing home placement and mortality. Neurology 1996;47:166-177.

13. Wimo A, Karlsson G, Nordberg A, Winblad B. Treatment of Alzheimer disease with tacrine: a cost-analysis model. Alzh Dis Assoc Disord 1997;11:191-200.

14. Rogers SL, Friedhoff LT, the Donepezil Study Group. The efficacy and safety of donepezil in patients with Alzheimer's disease: results of a US multicenter, randomized, double-blind, placebo-controlled trial. Dementia 1996;7:293-303.

15. Rogers SL, Farlow MR, Doody RS, Mohs R, Friedhoff LT, the Donepezil Study Group. A 24-week, double-blind, placebocontrolled trial of donepezil in patients with Alzheimer's disease. Neurology 1998;50:136-144.

16. Anand R, Gharabawi G. Clinical development of Exelon ${ }^{\mathrm{TM}}$ (ENA-713): the ADENA® programme. J Drug Dev Clin Pract 1996;8:9-14. 
17. Anand R, Gharabawi G, Enz A. Efficacy and safety results of the early phase studies with Exelon ${ }^{\mathrm{TM}}$ (ENA-713) in Alzheimer's disease: an overview. J Drug Dev Clin Pract 1996;8:1-8.

18. Becker RE, Colliver JA, Markwell SJ, Moriearty PL, Unni LK, Vicari S. Effects of metrifonate on cognitive decline in Alzheimer disease: a double-blind, placebo-controlled, 6 month study. Alzh Dis Assoc Disord 1998;12:54-57.

19. Schneider LS, Forette F. Alzheimer's disease symptomatic drugs: tacrine. In Gauthier S (ed). Clinical diagnosis and management of Alzheimer's disease. London: Martin Dunitz, 1996: 221-237.

20. Poirier J, Delisle MC, Quirion R, et al. Apolipoprotein $\varepsilon 4$ allele as a predictor of cholinergic deficits and treatment outcome in Alzheimer disease. Proc Nat Acad Sci 1995;92:12260-12264.

21. Oddoze $\mathrm{C}$, Michel BF, Berthézène $\mathrm{P}$, Clavel $\mathrm{C}$, Lucotte $\mathrm{G}$. Apolipoprotein $\mathrm{E} \varepsilon 4$ allele predicts a positive response to tacrine in Alzheimer's disease. Alzh Reports 1998;1:13-16.

22. Farlow MR, Lahiri DK, Poirier J, Davignon J, Schneider L, Hui SL. Treatment outcome of tacrine therapy depends on apolipoprotein genotype and gender of the subjects with Alzheimer's disease. Neurology 1998;50:669-677.

23. Tang MX, Jacobs D, Stern Y, et al. Effect of oestrogen during menopause on risk and age at onset of Alzheimer's disease. Lancet 1996;348:429-432.

24. Birge SJ. The role of estrogen in the treatment of Alzheimer's disease. Neurology 1997;48(Suppl 7):36-41.

25. Henderson VW. The epidemiology of estrogen replacement therapy and Alzheimer's disease. Neurology 1997;48 (Suppl.7):27-35.

26. Kawas C, Resnick S, Morrison A, et al. A prospective study of estrogen replacement therapy and the risk of developing Alzheimer's disease: the Baltimore longitudinal study of aging. Neurology 1997;48:1517-1521.

27. Schneider LS, Farlow MR, Henderson VW, Pogoda JM. Effects of estrogen replacement therapy on response to tacrine in patients with Alzheimer's disease. Neurology 1996;46:1580-1584.

28. Morris JH. Alzheimer's disease. In Esiri MM, Morris JH (eds). The neuropathology of dementia. Cambridge: Cambridge Univ Press, 1997:70-121.

29. Goedert M. Neurofibrillary pathology of Alzheimer's disease. Neuroscientist 1997;3:131-141.

30. Yankner BA. New clues to Alzheimer's disease: unraveling the roles of amyloid and tau. Nature Med 1996;2:850-851.

31. Drachman DA, Leber P. Treatment of Alzheimer's disease: searching for a breakthrough, settling for less. N Engl J Med 1997;347:1245-1247.

32. Cummings JL, Cyrus PA, Bieber F, et al. Metrifonate treatment of the cognitive deficits of Alzheimer's disease. Neurology 1998;50:1214-1221.

33. Morris JC, Cyrus PA, Orazem J, et al. Metrifonate benefits cognitive, behavioral, and global function in patients with Alzheimer's disease. Neurology 1998;50:1222-1230.

34. Kaufer DI, Cummings JL, Christine D. Effect of tacrine on behavoral symptoms in Alzheimer's disease: an open-label study. J Geriatr Psychiatry Neurol 1996;9:1-6. 